This study investigated whether aging was associated with epigenetic changes of DNA hypermethylation on immune gene expression and lymphocyte differentiation. We screened CG sites of methylation in blood leukocytes from different age populations, picked up genes with agerelated increase of CG methylation content more than $15 \%$, and validated immune related genes with CG hypermethylation involved in lymphocyte differentiation in the aged population. We found that 12 genes were associated with promoter or exon one DNA hypermethylation in the aged group. Two genes, GSTM1 and LMO2, were chosen to validate its aging-related CG hypermethylation in different leukocytes. Modulation of aging-associated GSTM1 methylation may be able to promote Th1 immunity in the elders.

\section{HIGHER FREQUENCY OF MICRONUCLEUS IS ASSOCIATED WITH SARCOPENIA IN AN ELDERLY FREE-LIVING COMMUNITY}

G.M. SilvaTavares ${ }^{1,2}$, V. Manfredini ${ }^{1}$, J. Mezzomo ${ }^{1}$, A.A. Güllich ${ }^{1}$, I. da Cruz ${ }^{3}$, M.G. Gottlieb², 1. physiotherapy, Federal University of Pampa, Uruguaiana, Rio Grande do Sul, Brazil, 2. Pontifícia Universidade Católica do Rio Grande do Sul, Porto Alegre, Rio Grande do Sul, Brazil, 3. Universidade Federal de Santa Maria, Santa Maria, Rio Grande do Sul, Brazil

Micronuclei is considered an important biomarker to monitor the health of individuals or populations exposed to different biological and environmental stressors. The aim of this present study was to verify the frequency of micronuclei lesions and the association with, muscle mass, lifestyle and health status in elderly residents in a free-living community. A cross-sectional observational investigation was performed on a sample of 168 elderly (60 males, 108 females) residents of a free-living community were included in this study. The blood sample was collected for a micronuclei assay. Social demographics, muscle mass, lifestyle and health status were also evaluated. The sample was categorized in two groups: lower micronucleus frequency (LM, $<5$ micronuclei/1000 nuclei) and higher micronucleus frequency (HM, $\geq 5$ micronuclei/1000 nuclei). In the percentile distribution, $50 \%$ of the sample presented $\geq 5$ micronuclei/1000 nuclei (the HM group). The mean age of sample was $68.41 \pm 6.13$. The mean micronucleus frequency was $5.87 \pm 2.75$ micronuclei/1000 nuclei. Only smoking habit $(\mathrm{p} \leq 0.002)$ and sarcopenia $(\mathrm{p} \leq 0.021)$ were significantly associated with higher micronucleus frequency. Higher frequency of micronucleus is associated with sarcopenia, independent of sex, age and smoking habit in an elderly sample.

\section{SESSION 930 (POSTER)}

\section{CANCER}

\section{EFFECTIVENESS OF ADJUVANT CHEMOTHERAPY FOR OLD PATIENT (70 YEARS OR OLDER) IN STAGE III COLON CANCER}

K. Yi-Hung, Y. Yu-Wen, H. Jui-Chu, Chang Gung Memorial Hospital, Chiayi, Taiwan, Chiayi, Taiwan
Background Surgery and postoperative adjuvant chemotherapy has been recommended for stage III colon cancer, especially in younger patients. In old patients (aged 70 years or older), the benefit is not so clear.

Methods This study was a retrospective observation of patients with stage III colon cancer who underwent curative surgery between January 1995 and December 2004 at the Chang Gung Memory Hospital. The disease-free (DFS) and cancer-specific survivals (CSS) of the old patients were calculated using univariate analysis with the Kaplan-Meier method and compared using the log-rank test. The Cox regression model was used for multivariate analysis for the confounding factors.

Results A total of 645 patients with stage III colon cancer were initially enrolled and stratified by age $(\geq 70,70>$ and $\geq 50$, younger than 50). The 50.5, 80.6 and 85.0 percent of three groups received adjuvant chemotherapy after curative surgery. No significant difference existed in 5-year CSS between old patients receiving adjuvant chemotherapy $(n=$ $108)$ and those who had surgery along $(n=106)(72.2$ vs. $65.2 \%, P=0.323$, Fig. 1A), whereas adjuvant chemotherapy improved 5-year DFS (54 vs. $48.8 \%, P=0.039$, Fig. 1B). However, multivariate analysis for 5-year DFS and CSS found an independent benefit for adjuvant chemotherapy in old patients with stage III colon cancer (in DFS, hazard ratio, $0.582,95 \%$ confidence interval, $0.394-0.860, P=0.007$; in CSS, hazard ratio, $0.035,95 \%$ confidence interval, $0.335-$ $0.960, \mathrm{P}=0.035)$.

Conclusions A multimodal approach in old patients with stage III colon cancer improved oncological outcomes.

\section{OUTCOMES AFTER BREAST CANCER SURGERY IN NURSING HOME RESIDENTS: A NATIONAL STUDY} V.L. Tang ${ }^{1,2}$, R. Sudore ${ }^{1,2}$, K.E. Covinsky ${ }^{1,2}$, C.S. Ritchie ${ }^{1,2}$, S. Zhao ${ }^{1}$, E. Finlayson ${ }^{1}$, 1. Medicine/Division of Geriatric Medicine; Division of Hospital Medicine, University of California San Francisco, San Francisco, California, 2. San Francisco VA Medical Center, San Francisco, California

Background: Over $60 \%$ of cancer-related operations in nursing-home residents occur for breast cancer. We studied functional and mortality outcomes after breast cancer surgery in nursing-home women.

Methods: We identified long-term stay nursing-home residents age $\geq 65$ who underwent inpatient breast cancer surgery in 2003-2009 using Medicare and Minimum DataSet (MDS). We examined 30-day mortality and hospital re-admission rates, stratified by procedure (lumpectomy, mastectomy, lymph node dissection with lumpectomy or mastectomy [LND]). In multivariate analysis, we examined factors associated with 1-year mortality. Functional status was measured by assessing the degree of dependence in seven activities of daily living (ADL): MDS-ADL scale with a 2 point difference as clinically significant.

Results: We identified 4,180 subjects (age: $82 \pm 7,48 \%$ dementia). Residents experienced significant functional decline that persisted 1 year after surgery (MDS-ADL score: lumpectomy: -3.4 , mastectomy: -2.9 , and LND: -2.5 ). Thirtyday readmission and mortality were high after surgery: lumpectomy: $26 \%$ and $9 \%$, mastectomy: $14 \%$ and $4 \%$, and LND: $15 \%$ and $2 \%$, respectively. One-year all-cause mortality was high: lumpectomy: $42 \%$, mastectomy: $31 \%$, and 
LND: $26 \%$. In a multivariate analysis, poor baseline MDSADL score before surgery was strongly associated with 1-year mortality-- lumpectomy: HR 2.6 (95\% CI:1.5-4.4), mastectomy: HR 2.1 (95\% CI:1.5-2.9), and LND: HR 1.9 (95\% CI:1.5-2.5).

Conclusions: Among nursing-home women residents who undergo breast cancer surgery, 30-day hospital readmission and mortality are high, as is 1-year all-cause mortality. Poor baseline function prior to surgery was strongly associated with 1-year mortality. Individualized goal-oriented care (i.e., hormonal therapy or symptom management only) should be considered.

\section{ASSOCIATION BETWEEN FRAILTY AND READMISSION AFTER GASTRECTOMY IN OLDER PATIENTS WITH GASTRIC CANCER}

Y. Choe, J. Joh, Y. Kim, Chonnam National University Hwasun Hospital, Hwasun, Chonnam, Korea (the Republic of)

Background: The incidence of gastric cancer in older people has been increased. Because older patients are at increased risk of postoperative complications and mortality, preoperative risk assessment in this population is important. In this study, we want to know if preoperative assessment of frailty could be useful for predicting the postoperative outcome in gastric cancer patients

Methods: We investigated 223 patients (136 men and 87 women) over 65 years old underwent gastric cancer surgery from April 2012 to March 2015. We used the Study of Osteoporotic Fractures (SOF) frailty index to assess the frailty. In order to find the predicting factors for readmission within 1-year of discharge after gastrectomy, we used logistic regression model.

Results: Total $26(11.7 \%)$ patients readmitted within 1-year after gastrectomy. Patients in "robust" group had a readmission rate of $4.4 \%$ and $19.1 \%$ in "pre-frail and frail" group. After adjusting age, gender, Eastern Cooperative Oncology Group (ECOG) performance statue (score $\geq 1$ ), histological type and stage (III, IV), the frailty (pre-frail and frail) was revealed predicting factor for readmission within 1-year of discharge after gastrectomy (Odds Ratio (OR) 5.74, 95\% Confidence Interval (CI) 1.78-18.48, p=0.003).

Conclusion: Preoperative risk assessment including frailty evaluation can predict the readmission within 1-year of discharge after gastrectomy. Thus, frailty assessment can help physicians to identify the risk and inform patients and their families of the risk for better decision making process in gastric cancer treatment

\section{FACTORS CONTRIBUTING TO FALLS IN OLDER BREAST CANCER SURVIVORS}
J. Blackwood, M. Huang, E. Neuman, M. Godoshian, L. Pfalzer, Physical Therapy, University of Michigan--Flint, Linden, Michigan

INTRODUCTION: In older adults, cancer and its treatment affect multiple body systems, leading to functional impairments, limited balance and impairments in walking, and thus contributing to an increased falls risk. The purpose of this study was to examine the health-related factors (physical, cognitive) contributing to falls in breast cancer survivors who were actively being treated (Active) versus those who were not receiving active treatment for their cancer (Non-Active).

METHODS: This cross-sectional study used data from the Surveillance, Epidemiology and End Results national cancer registry and Medicare Health Outcomes Survey linkage. Forward stepwise logistic regression analyses were completed to assess the contribution of health-related variables (difficulty walking or getting out of a chair, balance problems, and cognitive function) to falls in older breast cancer survivors.

RESULTS: Sample included 8187 cases. In the Active group ( $n=4045$ ), $27 \%$ reported falling and $40 \%$ had balance/ walking difficulties while those numbers were $35 \%$ and $24 \%$ respectively in the Non-Active group $(n=4142)$. Impaired cognitive function (difficulty with memory/concentration) was present in 18\% (Active) and in 13\% (Non-Active). Logistic regression modeling indicated that difficulties with memory/concentration and balance problems were significantly associated with falls in the Active group. In the NonActive group the presence of balance problems was the only significant variable contributing to falls $(p<0.01)$.

CONCLUSIONS: In older breast cancer survivors under active treatment for cancer, cognitive function and balance/ walking problems were both significantly associated with falls. Future research needs to delineate the impact of cancerspecific factors on functional mobility.

\section{HEALTH CARE PROFESSIONALS' PERSPECTIVES OF CARING FOR PATIENTS WITH HEPATOCELLULAR CARCINOMA}

L. Hansen, S. Rosenkranz, A. Schroeder, W. Naugler, Oregon Health \& Science University, Portland, Oregon

Background: Hepatocellular carcinoma (HCC) is the second leading cause of cancer related mortality in the world. It is often diagnosed at an advantaged stage. Due to the numerous physical and psychological symptoms and the number of treatments patients receive, management is extremely complex and may best be done by a multi-disciplinary liver tumor board. Research is lacking on these tumor boards and on health care professionals' (HCPs) perspectives on the challenges they face in caring for patients with HCC.

Aim: To examine challenges in symptom management, treatment therapies, and care of patients with HCC from the perspectives of urban and rural HCPs.

Methods: The study used a prospective, descriptive design. Semi-structured interview data were obtained from $10 \mathrm{HCPs}$ employed at an urban hospital, and 8 at urban and 8 at rural primary health care clinics. Data were analyzed using conventional content analysis.

Results: Tumor boards are a trustworthy and valuable resource but high number of referrals, access to treatment, and lines of communication are barriers to care. Lack of knowledge, expertise, and specialties make it difficult for rural HCPs to manage and coordinate the care for patients. Lack of follow-up care with primary care providers, inability by specialists to manage non-cancerous issues, and lack of continuity of care jeopardize symptom management.

Conclusions: Focus should be on improving communication between referring providers, patients, and multi-disciplinary care groups. Referral to palliative care should happen early in the treatment process. Early screening and regular 